\title{
Thermophysics of alkali and related azides II. Heat capacities of potassium, rubidium, cesium, and thallium azides from 5 to $350 \mathrm{~K}^{a, b}$
}

\author{
ROBERT W. CARLING ${ }^{c, d}$ and EDGAR F. WESTRUM, JR. ${ }^{e}$ \\ Department of Chemistry, University of Michigan, Ann Arbor, \\ Michigan 48109, U.S.A.
}

(Received 26 July 1977; in revised form 7 April 1978)

\begin{abstract}
The heat capacities of potassium, rubidium, cesium, and thallium azides were determined from 5 to $350 \mathrm{~K}$ by adiabatic calorimetry. Although the alkali-metal azides studied in this work exhibited no thermal anomalies over the temperature range studied, thallium azide has a bifurcated anomaly with two maxima at $(233.0 \pm 0.1) \mathrm{K}$ and $(242.04 \pm 0.02) \mathrm{K}$. The associated excess entropy was $0.90 \mathrm{cal}_{\mathrm{th}} \mathrm{K}^{-1} \mathrm{~mol}^{-1}$. The thermal properties of the azides and the corresponding structurally similar hydrogen difluorides are nearly identical. Both have linear symmetrical anions. However, thallium azide shows a solid-solid phase transition not exhibited by thallium hydrogen difluoride. At $298.15 \mathrm{~K}$ the values of $C_{p}^{\circ}, S^{\circ}$, and $-\left\{G^{\circ}(T)-H^{\circ}(0)\right\} / T$, respectively, are $18.38,24.86$, and $12.676 \mathrm{cal}_{\mathrm{th}} \mathrm{K}^{-1} \mathrm{~mol}^{-1}$ for potassium azide; $19.09,28.78$, and $15.58 \mathrm{cal}_{\mathrm{th}} \mathrm{K}^{-1} \mathrm{~mol}^{-1}$ for rubidium azide; $19.89,32.11$, and $18.17 \mathrm{cal}_{\mathrm{th}} \mathrm{K}^{-1} \mathrm{~mol}^{-1}$ for cesium azide; and 19.26, 32.09, and $18.69 \mathrm{cal}_{\mathrm{th}} \mathrm{K}^{-1} \mathrm{~mol}^{-1}$ for thallium azide. Heat capacities at constant volume for $\mathrm{KN}_{3}$ were deduced from infrared and Raman data.
\end{abstract}

\section{Introduction}

An interesting precedent to the study ${ }^{(1)}$ of alkali-metal and thallium azides is the longstanding interest in this laboratory in the orientational disorder of hydrogen difluoride anion, $\mathrm{HF}_{2}^{-}$in crystals. ${ }^{(2,3)}$ The isostructural compounds with linear anions $-\mathrm{KN}_{3}$, $\mathrm{RbN}_{3}, \mathrm{CsN}_{3}$, and $\mathrm{TIN}_{3}-$ crystallize in distorted CsCl-like structures as do the corresponding hydrogen difluorides. The distortion can be visualized by substituting an azide ion for a chloride ion in $\mathrm{CsCl}$ thereby expanding the structure in directions normal to the c-axis. Thus, a tetragonal unit cell is formed with space group, I4/ $m c m-D_{4 h^{*}}^{18}(4)$

At elevated temperatures, the tetragonal structure of the alkali-metal azides studied in this research can be expected to transform to a cubic $\mathrm{CsCl}$-like structurc due to

${ }^{a}$ For Part I see reference 1 .

${ }^{b}$ This work has been supported in part by the Chemical Thermodynamics Program, Chemistry Division, National Science Foundation under Contract Nos. GP-42525X and CHE-77-10049.

c Based in part upon a dissertation submitted to the Horace H. Rackham School of Graduate Studies at the University of Michigan in partial fulfillment of the requirements for the Ph.D. degree by R.W.C.

${ }^{d}$ Present address: Sandia Laboratories, Division 8313, Livermore, CA 94550, U.S.A.

"To whom correspondence concerning this paper should be directed. 
thermal disorder of the azide ions. Evidence of cubic high-temperature phases of $\mathrm{CsN}_{3}$ and $\mathrm{RbN}_{3}$ occurring at 424 and $588 \mathrm{~K}$, respectively, has been noted. ${ }^{(5)}$ The transition temperature increases with decreasing cation radius and hence the corresponding transition for $\mathrm{KN}_{3}$ would be expected to occur at approximately $748 \mathrm{~K}$, well above the melting temperature of $\mathrm{KN}_{3}$. The high-temperature behavior of the alkalimetal azides is supported by the fact that similar behavior is found in the corresponding alkali-metal hydrogen difluorides. ${ }^{(5)}$ The highly ionic azides are relatively stable but do decompose, albeit not violently, at their melting temperatures. $\Lambda$ s the bonding becomes more covalent the azides become highly explosive and can be detonated by heat, light, shock, and radiation. ${ }^{(7)}$

Pistorius $^{(8)}$ recently suggested - on the basis of extrapolations of $T(p)$ equilibrium lines assuming negligible curvature-that $\mathrm{RbN}_{3}$ and $\mathrm{CsN}_{3}$ at low pressure transform to low-temperature phases near 73 and $143 \mathrm{~K}$, respectively. This would be in contrast to the behavior of the corresponding alkali-metal hydrogen difluorides. ${ }^{(9)}$

$\mathrm{TIN}_{3}$ is known to transform-analogously to $\mathrm{RbN}_{3}$ and $\mathrm{CsN}_{3}$ - to a high-temperature cubic modification. Kezer and Rosenwasser ${ }^{(10)}$ studied this transformation by d.t.a. and reported a transition temperature of $564 \mathrm{~K}$. The reports of low-temperature transitions in $\mathrm{TIN}_{3}$ are numerous. ${ }^{(4,8,11-14)}$ The lowest-temperature phase of $\mathrm{TIN}_{3}$ is reported to be orthorhombic; ${ }^{(12)}$ a corresponding phase is not found in $\mathrm{TlHF}_{2},{ }^{(9)}$

Low-temperature adiabatic calorimetry permitted determination of the heat capacities of these azides, the energetics of the transition in $\mathrm{TIN}_{3}$, and also whether $\mathrm{RbN}_{3}$ and $\mathrm{CsN}_{3}$ undergo the low-temperature phase changes predicted by Pistorius. ${ }^{(8)}$

\section{Experimental}

\section{SAMPLE PREPARATION AND CHARACTERIZATION}

$\mathrm{KN}_{3}, \mathrm{RbN}_{3}$, and $\mathrm{CsN}_{3}$. The preparation of each of these azides closely resembles the preparation of the same substances by Suhrmann and Clusius. ${ }^{(15)}$ Optical-grade potassium, rubidium, and cesium carbonates were purchased from Electronic Space Products, Inc., Los Angeles, CA. In a Teflon receiving beaker, $50 \mathrm{~g}$ of the desired alkali metal carbonate were dissolved in about $300 \mathrm{~cm}^{3}$ of water. Excess $\mathrm{NaN}_{3}$, in the form of an aqueous slush, was put in a round-bottom flask to which a spray trap and condenser were fused. The flask was gently warmed to insure complete dissolution of the $\mathrm{NaN}_{3}$. To the solution, dilute sulfuric acid (about 12 per cent by mass) was added dropwise from a separatory funnel. The hydrogen azide thus formed distilled into the Teflon receiving beaker, liberated $\mathrm{CO}_{2}$, and formed the desired alkali-metal azide. The distillation process was continued about $2 \mathrm{~h}$ to ensure complete removal of carbon dioxide from the carbonate solution. This procedure was repeated until an adequate amount of each azide was prepared.

Recrystallization of each azide from an aqueous solution was achieved by dissolving the respective azide in water, filtering the solution to remove any insoluble impurities, and then gently heating it until incipient precipitation occurred. After cooling the solution to $300 \mathrm{~K}$, we added a two-fold excess of pure ethanol to enhance precipitation. The precipitated azide was filtered, washed with ethanol, and dried for several hours at $380 \mathrm{~K}$. Each azide was recrystallized three times. $\mathrm{RbN}_{3}$ was heated only in the 
drying oven and was later found to contain trapped solvent. Further details of this determination are postponed until after the presentation of calorimetric results. However, after $2 \mathrm{~h}$ in the drying oven, $\mathrm{CsN}_{3}$ and $\mathrm{KN}_{3}$ were crushed to uniform size in an agate mortar and placed in a vacuum desiccator over $\mathrm{P}_{2} \mathrm{O}_{5}$. The dessicator was evacuated for about $4 \mathrm{~h}$ through a liquid-nitrogen trap. The samples of $\mathrm{CsN}_{3}$ and $\mathrm{KN}_{3}$ were then removed from the desiccator, placed back in the drying oven for about $4 \mathrm{~h}$, and subsequently stored in a desiccator over $\mathrm{P}_{2} \mathrm{O}_{5}$.

X-ray diffraction analyses of $\mathrm{KN}_{3}, \mathrm{RbN}_{3}$, and $\mathrm{CsN}_{3}$ yielded lattice parameters which agree well with those of previous investigations. A comparison of the lattice parameters of these and other azides have been reported previously ${ }^{(16)}$ and is available in a supplementary document. ${ }^{(17)}$

The azide content of $\mathrm{RbN}_{3}$ was measured by hydrogen-ion titration after oxidation of the azide ion by nitrite. ${ }^{(18)}$ Although a reasonably good result was obtained by this method (mass per cent azide indicated $39.90 \pm 0.05$; theoretical: 39.96 ), this method was abandoned and a more straightforward analysis was undertaken for the azide content of potassium and cesium azides. The azide content was determined graviInetrically by weighing silver azide. Samples of each azide were placed in a beaker and dissolved in $75 \mathrm{~cm}^{3}$ of 8 per cent by mass potassium nitrate solution. Silver nitrate $\left(0.35 \mathrm{~mol} \mathrm{dm}^{-3}\right)$ was added dropwise to each sample until complete precipitation occurred; the beakers were then stored overnight in the dark. Subsequently the precipitates were filtered in subdued light, rinsed with water, and dried to constant mass in the oven at $380 \mathrm{~K}$. The elemental analysis indicated $(51.82 \pm 0.02)$ mass per cent $\mathrm{N}_{3}$ for $\mathrm{KN}_{3}$ and (24.03 \pm 0.02$)$ mass per cent $\mathrm{N}_{3}$ for $\operatorname{Cs} \mathrm{N}_{3}$ (theoreical: 51.80 and 24.02, respectively).

TIN $_{3}$. High-purity thallium was purchased in the form of rods from American Smelting and Refining Company, Central Research Laboratories, South Plainfield, New Jersey. To prepare thallium turnings, the $6 \mathrm{~mm}$ diameter rods were first rinsed in concentrated nitric acid to remove any oxide layer present. The turnings generated by "sharpening the rods" in a pencil sharpener were collected in a shallow Teflon dish containing pure ethanol. The turnings were transferred to a Teflon thimble in a Soxhlet apparatus, where they were held in refluxing ethanol for several hours until most of the thallium had reacted. To the ethanol solution a volume of water equal to that of the ethanol was added. The yellow thallium hydroxide precipitate that formed upon addition of the water was filtered and dissolved in warm water. Carbon dioxide, purchased from Matheson and labelled "bone dry", was then bubbled into the thallium hydroxide solution whereupon a white flocculent $\mathrm{Tl}_{2} \mathrm{CO}_{3}$ precipitate formed. This precipitate was filtered, washed with cold water, and then dried at $380 \mathrm{~K}$ for several hours.

Before precipitation of $\mathrm{TIN}_{3}$, both $\mathrm{Tl}_{2} \mathrm{CO}_{3}$ and $\mathrm{NaN}_{3}$ were recrystallized from water. Each solid was dissolved in water and the two solutions were combined whereupon a yellow precipitatc of thallium azide immediately formed. The precipitate was filtered, washed with distilled water and pure ethanol, and then dried at $380 \mathrm{~K}$.

The $\mathrm{TIN}_{3}$ thus prepared was to be recrystallized from water (in which it is not very soluble $)^{(19)}$ so solvent was continually added while heating until total dissolution occurred. The solution was then filtered while still hot to remove insoluble impurities 
and allowed to cool slowly to $300 \mathrm{~K}$ while long acicular yellow crystals formed. These crystals were filtered, washed with pure ethanol and then dried for $6 \mathrm{~h}$ at $380 \mathrm{~K}$. The recrystallization process was repeated three times and the product stored in a desiccator over $\mathrm{CaSO}_{4}$.

X-ray diffraction analysis of $\mathrm{TIN}_{3}$ yielded lattice parameters which are in excellent accord with those of other investigators. ${ }^{(16,17)}$ The thallium content was determined by a volumetric titration with standard potassium iodate solution as the titrant. ${ }^{(20)}$ The azide solution required gentle warming completely to dissolve the $\mathrm{TlN}_{3}$ before analysis. Elemental analysis indicated $(82.92 \pm 0.02)$ mass per cent $\mathrm{Tl}$ (theoretical: 82.94). Further analyses of the sample were performed after the heat-capacity determinations were completed and will be discussed later.

Loading details. Heat-capacity measurements for all four samples were made in the Mark II adiabatic cryostat. ${ }^{(1,21)}$ Loading information for these samples is given in table 1 .

TABLE 1. Calorimeter loading information

\begin{tabular}{lcccccc}
\hline Compound & $\begin{array}{c}\text { Calorimeter } \\
\text { no. }\end{array}$ & $\frac{V}{\mathrm{~cm}^{3}}$ & $\frac{M^{a}}{\mathrm{~g} \mathrm{~mol}^{-1}}$ & $\frac{m}{\mathrm{~g}}$ & $\frac{p(\mathrm{He})}{\mathrm{kPa}}$ & $\frac{\rho^{b}}{\mathrm{~g} \mathrm{~cm}^{-3}}$ \\
\hline $\mathrm{KN}_{3}$ & $\mathrm{~W}-52$ & 59.11 & 81.118 & 50.2650 & 6.6 & 2.032 \\
$\mathrm{RbN}_{3}$ & $\mathrm{~W}-28$ & 92.31 & 127.487 & $84.8699^{\circ}$ & 5.7 & 2.825 \\
$\mathrm{CsN}_{3}$ & $\mathrm{~W}-52$ & 59.11 & 174.922 & 46.1975 & 7.6 & 3.384 \\
$\mathrm{TlN}_{3}$ & $\mathrm{~W}-52$ & 59.11 & 246.3901 & $89.3849^{\mathrm{c}}$ & 6.5 & 5.769 \\
\hline
\end{tabular}

${ }^{a}$ Based on 1968 IUPAC scale of atomic wcights.

${ }^{b}$ Crystallographic density from lattice parameters of this research, four molecules per unit cell.

c Mass corrected for traces of water present.

\section{Results}

\section{HEAT CAPACITY VALUES FOR $\mathrm{KN}_{3}, \mathrm{RbN}_{3}$, AND CsN}

Heat capacities in chronological sequence at the mean temperatures of determination are presented in tables 2 and 3. Approximate temperature increments can usually be inferred from adjacent mean temperatures. These results have been adjusted for curvature and are considered to have a probable error of about 5 per cent at $5 \mathrm{~K}$, decreasing to 1 per cent at $10 \mathrm{~K}$, and to less than 0.1 per cent above $20 \mathrm{~K}$. The values are based upon IPTS-48. Results from several series of determinations taken to ascertain the enthalpy increment through the bifurcated anomaly in $\mathrm{TIN}_{3}$ have been summarized in table 4.

Molar heat-capacity curves for $\mathrm{KN}_{3}, \mathrm{RbN}_{3}$, and $\mathrm{CsN}_{3}$ are displayed in figure 1. No thermal anomalies due to structural changes in these compounds were observed; however, the heat capacity of $\mathrm{RbN}_{3}$ revealed a small bump located at $(257.3 \pm 0.8) \mathrm{K}$ which was believed to result from the fusion of water trapped in the sample. To test this interpretation, the freezing temperature of a saturated $\mathrm{RbN}_{3}$ aqueous solution was determined with a Hewlett-Packard quartz thermometer as $257.5 \mathrm{~K}$ and supports the interpretation of the anomaly as due to residual water trapped in the $\mathrm{RbN}_{3}$ 
TABLE 2. Heat capacities of $\mathrm{KN}_{3}, \mathrm{RbN}_{3}$, and $\mathrm{CsN}_{3}$

$$
\left(\mathrm{cal}_{\mathrm{th}}=4.184 \mathrm{~J}\right)
$$

\begin{tabular}{|c|c|c|c|c|c|c|c|}
\hline $\begin{array}{l}T \\
\mathrm{~K}\end{array}$ & $\frac{C_{p}}{\mathrm{cal}_{\mathrm{th}} \mathrm{K}^{-1} \mathrm{~mol}^{-1}}$ & $\begin{array}{l}T \\
\mathrm{~K}\end{array}$ & $\frac{C_{p}}{\mathrm{cal}_{\mathrm{th}} \mathrm{K}^{-1} \mathrm{~mol}^{-1}}$ & $\frac{T}{\mathrm{~K}}$ & $\frac{C_{p}}{\mathrm{cal}_{\mathrm{th}} \mathrm{K}^{-1} \mathrm{~mol}^{-1}}$ & $\begin{array}{ll}T \\
\mathrm{~K} & \mathrm{cal}_{\mathrm{L}}\end{array}$ & $\frac{C_{p}}{\mathrm{~K}^{-1} \mathrm{~mol}^{-1}}$ \\
\hline \multicolumn{8}{|c|}{$\mathrm{KN}_{3}$} \\
\hline \multicolumn{2}{|r|}{ Series I } & 347.39 & 19.43 & \multicolumn{2}{|r|}{ Series III } & $\begin{array}{l}23.99 \\
26.31\end{array}$ & $\begin{array}{l}1.012 \\
1.329\end{array}$ \\
\hline 209.54 & 16.16 & \multicolumn{2}{|r|}{ Series II } & 5.64 & 0.014 & 28.73 & 1.688 \\
\hline 215.15 & 16.34 & & & 7.07 & 0.023 & 31.44 & 2.137 \\
\hline 223.78 & 16.56 & 96.01 & 11.26 & 8.28 & 0.036 & 34.76 & 2.727 \\
\hline 233.99 & 16.83 & 103.34 & 11.80 & 9.38 & 0.053 & 38.69 & 3.456 \\
\hline 244.27 & 17.08 & 112.49 & 12.39 & 10.34 & 0.074 & 43.12 & 4.281 \\
\hline 254.43 & 17.34 & 121.94 & 12.93 & 11.39 & 0.089 & 48.08 & 5.194 \\
\hline 264.66 & 17.59 & 131.74 & 13.44 & 12.62 & 0.120 & 53.44 & 6.140 \\
\hline 275,45 & 17.84 & 141.94 & 13.90 & 12.52 & 0.119 & 58.85 & 7.025 \\
\hline 286.31 & 18.10 & 152.25 & 14.31 & 13.96 & 0.173 & 64.69 & 7.914 \\
\hline 296.79 & 18.34 & 162.66 & 14.71 & 15.29 & 0.238 & 70.70 & 8.707 \\
\hline 307.45 & 18.57 & 173.04 & 15.09 & 16.73 & 0.317 & 76.23 & 9.373 \\
\hline 318.30 & 18.81 & 183.20 & 15.40 & 18.35 & 0.434 & 81.99 & 10.02 \\
\hline 329.04 & 19.04 & 193.42 & 15.69 & 20.06 & 0.583 & 89.11 & 10.72 \\
\hline \multirow[t]{2}{*}{339.68} & 19.26 & 203.69 & 16.00 & 21.90 & 0.768 & 97.59 & 11.39 \\
\hline & & 213.79 & 16.29 & & & & \\
\hline \multicolumn{8}{|c|}{$\mathrm{RbN}_{3}$} \\
\hline \multirow{2}{*}{\multicolumn{2}{|c|}{ Series 1}} & 56.54 & 8.558 & 7.44 & 0.072 & 261.90 & 18.90 \\
\hline & & 63.72 & 9.599 & 8.14 & 0.086 & & \\
\hline 149.25 & 15.13 & 71.17 & 10.48 & 8.92 & 0.123 & \multicolumn{2}{|c|}{ Series VI } \\
\hline 151.62 & 15.23 & 77.96 & 11.19 & 9.72 & 0.171 & & \\
\hline 157.68 & 15.45 & 84.28 & 11.80 & 10.56 & 0.234 & 242.74 & 17.78 \\
\hline 166.99 & 15.75 & 91.21 & 12.34 & 11.48 & 0.270 & $\Delta H$ Detn. & \\
\hline 176.96 & 16.05 & 98.77 & 12.82 & 12.98 & 0.424 & 280.69 & 18.85 \\
\hline 187.02 & 16.34 & 107.01 & 13.30 & 13.96 & 0.545 & \multirow{2}{*}{\multicolumn{2}{|c|}{ Series VII }} \\
\hline 196.92 & 16.60 & 115.94 & 13.76 & 14.95 & 0.678 & & \\
\hline 206.88 & 16.87 & \multirow{2}{*}{\multicolumn{2}{|c|}{ Series III }} & 16.17 & 0.840 & & \\
\hline 216.91 & 17.14 & & & 17.59 & 1.061 & 243.18 & 17.78 \\
\hline 226.81 & 17.39 & & & 19.14 & 1.318 & $\Delta H$ Detn. & \\
\hline 236.78 & 17.65 & 103.92 & 13.12 & 20.93 & 1.644 & 268.04 & 18.44 \\
\hline 246.82 & 17.92 & 113.12 & 13.63 & 23.05 & 2.043 & \\
\hline 256.72 & & 121.95 & 14.06 & 25.62 & 2.566 & & \\
\hline 266.60 & 18.44 & 131.35 & 14.47 & 28.52 & 3.165 & \multicolumn{2}{|c|}{ Series VIII } \\
\hline 277.09 & 18.67 & 141.32 & 14.87 & 31.64 & 3.831 & 242.84 & 17.77 \\
\hline 287.47 & 18.92 & 151.03 & 15.22 & 35.12 & 4.584 & 247.56 & 17.94 \\
\hline 297.75 & 19.18 & 160.52 & 15.53 & 38.74 & 5.337 & 251.31 & 18.16 \\
\hline 308.25 & 19.42 & \multirow{2}{*}{\multicolumn{2}{|c|}{ Series IV }} & 44.07 & 6.399 & 253.64 & 18.29 \\
\hline 318.98 & 19.67 & & & 48.30 & 7.219 & 255.50 & 18.46 \\
\hline 329.60 & 19.90 & & & 53.17 & 8.025 & 257.28 & 20.08 \\
\hline 340.14 & 20.14 & 4.18 & 0.000 & 58.82 & 8.900 & 259.03 & 19.29 \\
\hline \multirow[t]{3}{*}{346.18} & 20.22 & 4.74 & 0.020 & \multirow{2}{*}{\multicolumn{2}{|c|}{ Series $Y$}} & 260.84 & 18.66 \\
\hline & \multirow{3}{*}{ Series II } & 5.27 & 0.020 & & & 262.69 & 18.46 \\
\hline & & 5.76 & 0.028 & & & 265.00 & 18.39 \\
\hline & & 6.26 & 0.037 & 242.81 & 17.82 & 268.69 & 18.49 \\
\hline 51.78 & 7.794 & 6.80 & 0.047 & 252.44 & 18.46 & & \\
\hline
\end{tabular}


TABLE 2-continued

\begin{tabular}{|c|c|c|c|c|c|c|c|}
\hline$\frac{T}{\mathrm{~K}}$ & $\frac{C_{p}}{K-1 \mathrm{~mol}-1}$ & $\frac{T}{K}$ & $\frac{C_{p}}{K-1}$ & $\frac{T}{K}$ & $\frac{C_{D}}{K-1}$ & $\frac{T}{K}$ & $\frac{C_{p}}{K-1}$ \\
\hline $\mathbf{K}$ & $\mathrm{cal}_{\mathrm{th}} \mathrm{K}^{-1} \mathrm{~mol}^{-1}$ & $\mathbf{K}$ & $\mathrm{cal}_{\mathrm{th}} \mathrm{K}^{-1} \mathrm{~mol}^{-1}$ & $\mathrm{~K}$ & $\mathrm{cal}_{\mathrm{th}} \mathbf{K}^{-1} \mathrm{~mol}^{-1}$ & $\mathbf{K}$ & $\mathrm{cal}_{\mathrm{th}} \mathrm{K}^{-1} \mathrm{~mol}^{-1}$ \\
\hline \multicolumn{8}{|c|}{$\mathrm{CsN}_{3}$} \\
\hline \multirow{2}{*}{\multicolumn{2}{|c|}{ Series I }} & \multirow{2}{*}{\multicolumn{2}{|c|}{ Series II }} & 204.93 & 17.29 & 16.27 & 1.808 \\
\hline & & & & 214.23 & 17.54 & 17.96 & 2.192 \\
\hline 215.77 & 17.60 & 66.07 & 11.06 & & & 19.82 & 2.615 \\
\hline 226.49 & 17.90 & 74.63 & 11.88 & \multicolumn{2}{|r|}{ Series III } & 21.88 & 3.079 \\
\hline 236.66 & 18.16 & 82.73 & 12.59 & & & 23.91 & 3.540 \\
\hline 247.37 & 18.44 & 91.14 & 13.20 & 4.56 & 0.020 & 26.00 & 4.022 \\
\hline 257.96 & 18.73 & 100.42 & 13.70 & 4.94 & 0.062 & 28.64 & 4.620 \\
\hline 268.42 & 19.01 & 110.50 & 14.18 & 5.36 & 0.076 & 32.02 & 5.381 \\
\hline 278.78 & 19.31 & 120.90 & 14.62 & 6.40 & 0.137 & 36.10 & 6.267 \\
\hline 289.00 & 19.62 & 131.20 & 15.02 & 7.32 & 0.207 & 40.50 & 7.165 \\
\hline 299.11 & 19.92 & 141.80 & 15.40 & 8.25 & 0.309 & 45.01 & 8.010 \\
\hline 309.67 & 20.23 & 152.73 & 15.76 & 9.25 & 0.456 & 49.64 & 8.841 \\
\hline 320.30 & 20.53 & 163.40 & 16.09 & 10.41 & 0.646 & 54.66 & 9.579 \\
\hline 330.48 & 20.83 & 173.85 & 16.41 & 11.75 & 0.857 & 59.78 & 10.29 \\
\hline 340.55 & 21.14 & 184.11 & 16.70 & 13.26 & 1.162 & 65.20 & 10.96 \\
\hline 347.55 & 21.36 & 194.46 & 17.00 & 14.75 & 1.490 & 71.76 & 11.63 \\
\hline
\end{tabular}

TABLE 3. Heat capacity of $\mathrm{TIN}_{3}$

$\left(\mathrm{cal}_{\mathrm{th}}=4.184 \mathrm{~J}\right)$

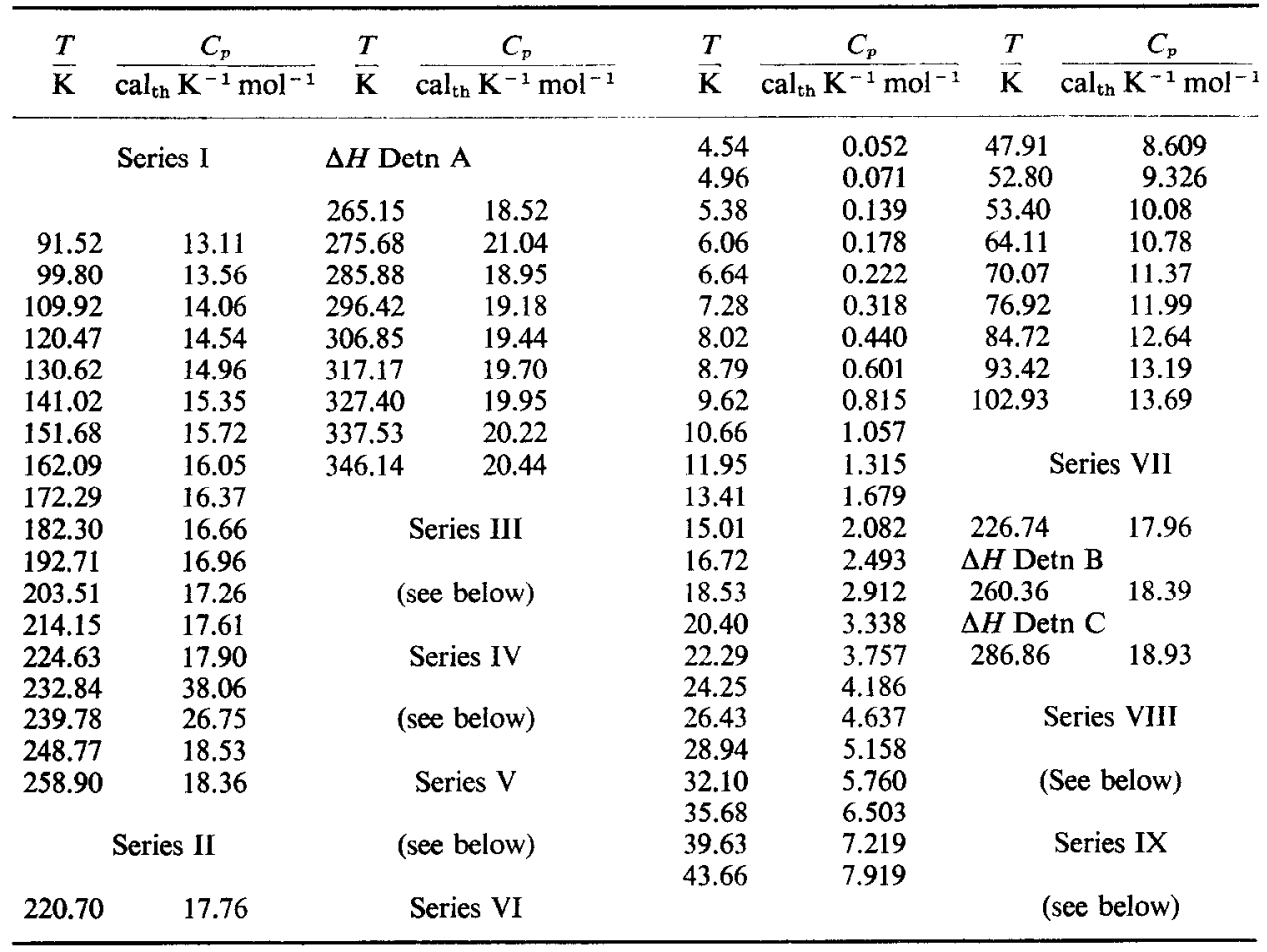


TABLE 3-continued

\begin{tabular}{|c|c|c|c|c|c|c|c|}
\hline$T$ & $\Delta T$ & $\left\langle C_{p}\right\rangle$ & $C_{p}$ & $T$ & $\underline{\Delta T}$ & $\left\langle C_{p}\right\rangle$ & $C_{p}$ \\
\hline$\overline{\mathbf{K}}$ & K & \multicolumn{2}{|c|}{$\mathrm{cal}_{\mathrm{th}} \mathrm{K}^{-1} \mathrm{~mol}^{-1} \mathrm{cal}_{\mathrm{th}} \mathrm{K}^{-1} \mathrm{~mol}^{-1}$} & $\overrightarrow{\mathrm{K}}$ & $\vec{K}$ & \multicolumn{2}{|c|}{$\mathrm{CaI}_{\mathrm{th}} \mathrm{K}^{-1} \mathrm{~mol}^{-1} \mathrm{cal}_{\mathrm{th}} \mathrm{K}^{-1} \mathrm{~mol}^{-1}$} \\
\hline \multirow{2}{*}{\multicolumn{4}{|c|}{ Series III }} & 274.37 & 0.91 & 19.00 & 18.85 \\
\hline & & & & 275.27 & 0.92 & 18.91 & 18.78 \\
\hline 228.77 & 4.20 & 18.10 & 18.04 & 276.84 & 2.28 & 18.69 & 18.76 \\
\hline 231.90 & 2.06 & 28.66 & 18.57 & \\
\hline 233.24 & 0.70 & 104.3 & 100.6 & & & Series V & \\
\hline 234.34 & 1.54 & 41.81 & 41.81 & & & & \\
\hline 236.18 & 2.17 & 26.68 & 26.57 & 227.62 & 4.71 & 18.02 & 18.02 \\
\hline 238.43 & 2.38 & 23.39 & 23.33 & \multicolumn{4}{|c|}{$\Delta H$ Detn $\mathrm{E}$} \\
\hline 240.74 & 2.26 & 25.20 & 24.53 & $\Delta H$ Detn & $F$ & & \\
\hline 242.83 & 1.94 & 31.00 & 30.75 & 270.30 & 1.98 & 19.28 & 19.28 \\
\hline 245.16 & 2.77 & 18.58 & 18.68 & 271.72 & 0.90 & 21.17 & 21.10 \\
\hline 247.94 & 2.79 & 18.37 & 18.37 & 272.48 & 0.67 & 32.27 & 26.90 \\
\hline 251.42 & 4.19 & 18.30 & 18.30 & 273.16 & 0.76 & 27.35 & 20.06 \\
\hline & & & & 274.01 & 0.97 & 19.00 & 18.94 \\
\hline & & Series IV & & 275.52 & 2.10 & 19.06 & 18.77 \\
\hline & & & 1803 & 277.62 & 2.12 & 18.73 & 18.73 \\
\hline $\begin{array}{l}228.22 \\
230.25\end{array}$ & $\begin{array}{l}3.55 \\
0.52\end{array}$ & 18.03 & $\begin{array}{l}18.05 \\
18.12\end{array}$ & \multirow{3}{*}{\multicolumn{4}{|c|}{ Series VIII }} \\
\hline 230.25 & 0.52 & 18.12 & $\begin{array}{l}18.12 \\
18.20\end{array}$ & & & & \\
\hline 230.77 & 0.52 & 18.22 & & & & & \\
\hline 231.28 & 0.52 & 18.25 & 18.34 & 240.45 & 2.03 & 24.17 & 23.96 \\
\hline 231.80 & 0.52 & 18.52 & 18.52 & 241.61 & 0.31 & 27.20 & 27.20 \\
\hline 232.31 & 0.51 & 18.82 & 18.86 & 241.83 & 0.13 & 35.28 & 35.28 \\
\hline 232.73 & 0.34 & 33.09 & 19.71 & 241.94 & 0.09 & 55.34 & 55.34 \\
\hline 233.00 & 0.30 & 123.2 & 123.2 & 242.00 & 0.04 & 118.6 & 118.6 \\
\hline 233.27 & 0.35 & 95.66 & 95.66 & 242.04 & 0.03 & 164.1 & 164.1 \\
\hline 233.66 & 0.50 & 64.63 & 64.63 & 242.08 & 0.07 & 76.53 & 76.53 \\
\hline 234.22 & 0.68 & 44.52 & 43.90 & 271.33 & 1.82 & 20.39 & 20.29 \\
\hline 234.96 & 0.86 & 33.28 & 33.28 & 272.29 & 0.18 & 24.58 & 24.26 \\
\hline 235.86 & 0.98 & 27.67 & 27.67 & 272.41 & 0.09 & 26.11 & 26.11 \\
\hline 236.87 & 1.06 & 24.94 & 24.94 & 272.49 & 0.08 & 26.95 & 27.78 \\
\hline 237.93 & 1.10 & 23.60 & 23.60 & 272.56 & 0.08 & 29.90 & 29.90 \\
\hline 239.03 & 1.12 & 23.20 & 23.20 & 272.62 & 0.07 & 32.13 & 32.13 \\
\hline 240.13 & 1.10 & 23.62 & 23.62 & 272.71 & 0.13 & 36.96 & 36.96 \\
\hline 241.19 & 1.04 & 25.59 & 25.59 & & & & \\
\hline 241.97 & 0.53 & 59.98 & 72.00 & \multirow{2}{*}{\multicolumn{4}{|c|}{ Series IX }} \\
\hline 242.48 & 0.50 & 24.00 & 24.00 & & & & \\
\hline 243.00 & 0.56 & 20.35 & 20.35 & 231.50 & 2.63 & 19.44 & 18.40 \\
\hline 243.94 & 1.32 & 19.17 & 19.17 & 232.84 & 0.10 & 25.61 & 25.61 \\
\hline 245.27 & 1.35 & 18.66 & 18.66 & 232.90 & 0.09 & 30.40 & 30.40 \\
\hline \multicolumn{4}{|c|}{$\Delta H$ Detn $\mathrm{D}$} & 232.94 & 0.08 & 35.44 & 35.44 \\
\hline 273.48 & 0.90 & 19.42 & 19.39 & 232.98 & 0.07 & 38.42 & 45.00 \\
\hline
\end{tabular}

${ }^{a}$ The symbol $\left\langle C_{p}\right\rangle$ represents mean values of the heat capacity as calculated directly from finite $\Delta H / \Delta T$ without curvature correction.

${ }^{b}$ The symbol $C_{p}$ in columns adjacent to $\left\langle C_{p}\right\rangle$ represents the value of the heat capacity read from the smoothed curve at temperature $T$. Elsewhere in the table it represents $C_{p}$ analytically corrected for curvature. 
TABLE 4. Enthalpy determinations for overlapping anomalies in $\mathrm{TIN}_{3}$ $\left(\mathrm{cal}_{\mathrm{th}}=4.184 \mathrm{~J}\right)$

\begin{tabular}{lcccc}
\hline \multicolumn{1}{c}{ Designation } & $\frac{T_{2}}{\mathrm{~K}}$ & $\frac{T_{2}}{\mathrm{~K}}$ & $\frac{H^{\circ}\left(T_{2}\right)-H^{\circ}\left(T_{1}\right)}{\mathrm{cal}_{\mathrm{th}} \mathrm{mol}^{-1}}$ & $\frac{H^{\circ}(261 \mathrm{~K})-H^{\circ}(220 \mathrm{~K})}{\mathrm{cal}_{\mathrm{th}} \mathrm{mol}^{-1}}$ \\
\hline A (Series II) & 225.91 & 259.52 & 804.93 & 937.63 \\
B (Series V) & 229.97 & 253.80 & 627.90 & 938.30 \\
E (Series VII) & 229.11 & 255.54 & 675.64 & 938.22 \\
& & & Mean: & $\frac{938.0 \pm 0.5}{742.1 \pm 2}$ \\
& & & Lattice: & $\frac{742.1 \pm 2}{195.9 \pm 2}$ \\
\hline
\end{tabular}

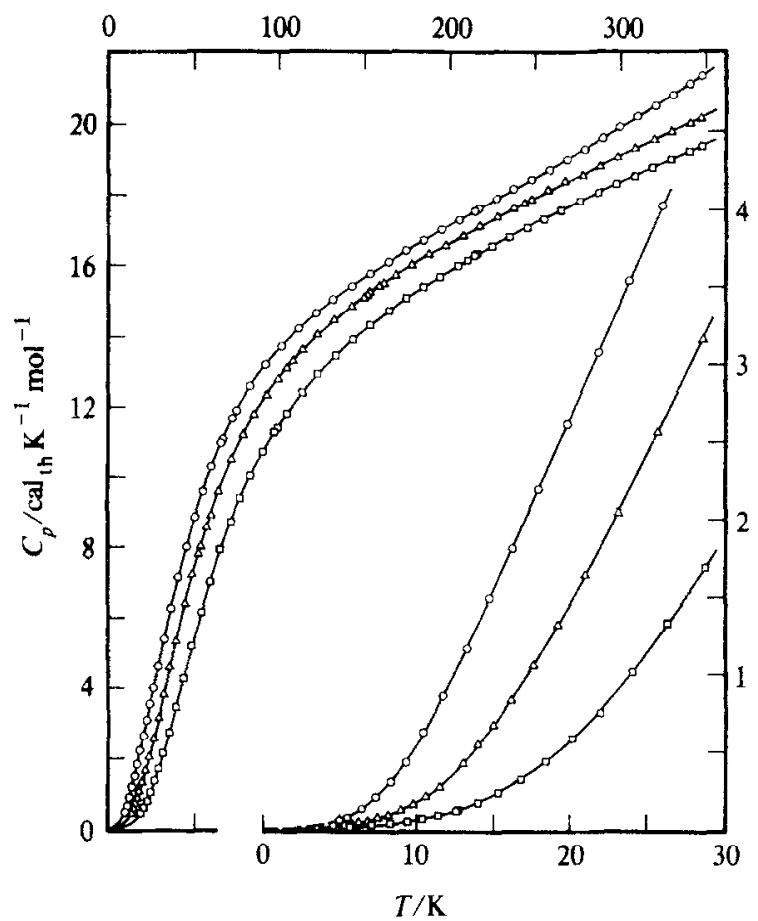

FIGURE 1. Experimental heat capacities: $L j, \mathrm{KN}_{3} ; \triangle, \mathrm{RbN}_{3} ; \circlearrowleft, \mathrm{CsN}_{3}$.

sample. From the excess enthalpy in the region of this small bump divided by the enthalpy of fusion of water, ${ }^{(22)}$ a value 0.07 mass per cent of $\mathrm{H}_{2} \mathrm{O}$ was computed.

\section{HEAT CAPACITY VALUES FOR TIN T $_{3}$}

The molar heat capacity of $\mathrm{TIN}_{3}$ displayed in figures 2 and 3 revealed a bifurcated anomaly with the two maxima cccurring at $(233.0 \pm 0.1)$ and $(242.04 \pm 0.02) \mathrm{K}$. In addition, a small bump at $272.7 \mathrm{~K}$ was thought to be due to the fusion of water in the sample. Iqbal and Malhotra ${ }^{(11)}$ noted a transition in the Raman spectra occurring on 


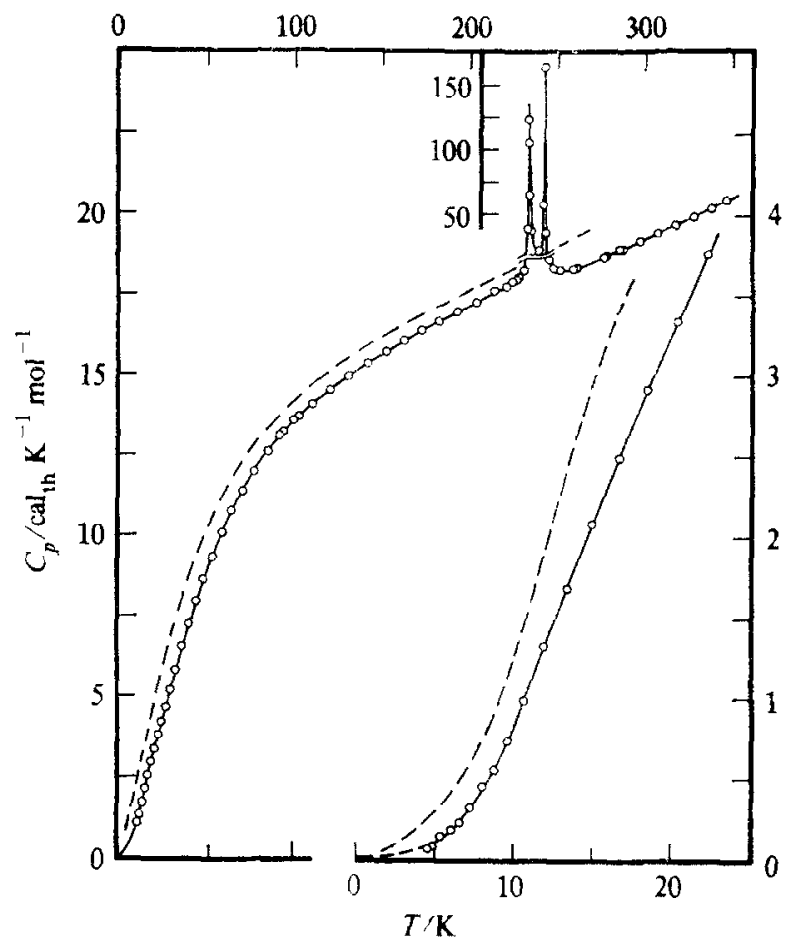

FIGURE 2. Experimental heat capacities: - - TIN $_{3}$ :- - , $\mathrm{TlHF}_{2}$.

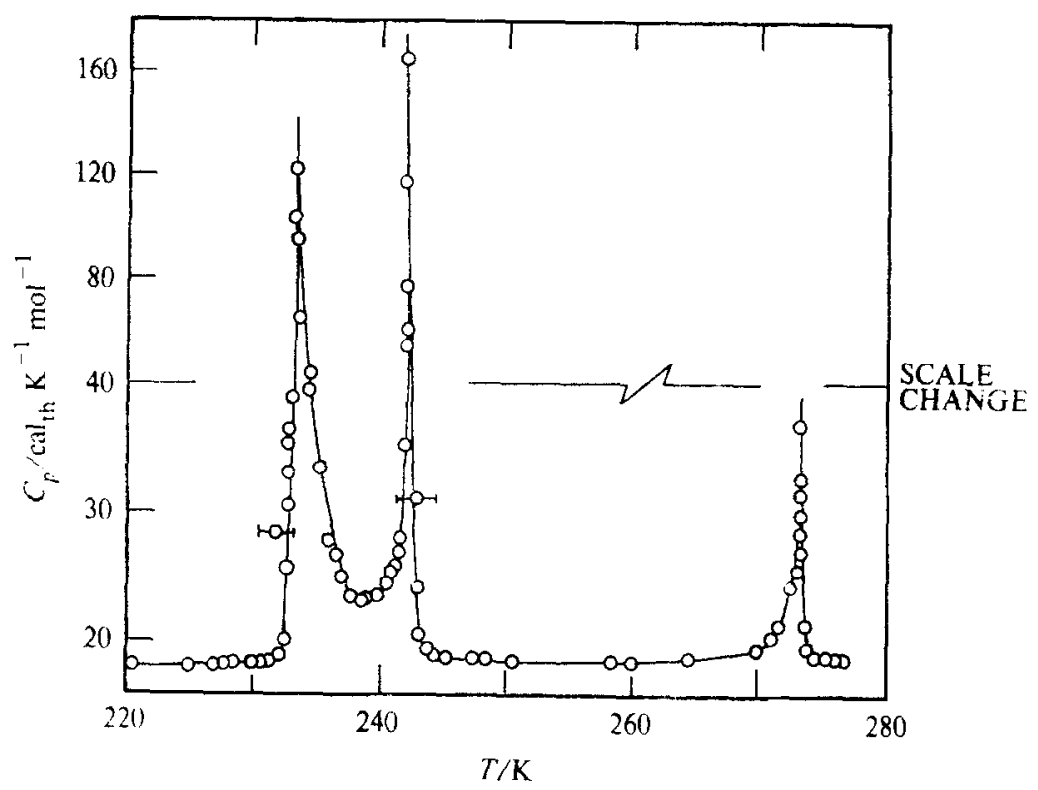

FIGURE 3. Experimental heat capacity of $\mathrm{TIN}_{3}$ in the region of the the two transitions and the thermal anomaly attributed to the presence of water. $O$, experimental values; $-\longrightarrow$, smoothed curve
(compare text). 
warming to $278 \mathrm{~K}$. On cooling the sample two of the spectral bands split into four bands at $269 \mathrm{~K}$ and persisted as low as $95 \mathrm{~K}$. Iqbal now considers that his previous interpretation of a phase change at $278 \mathrm{~K}$ is incorrect, ${ }^{(23)}$ and that his spectral result was due to sluggishness of the first-order transition near $240 \mathrm{~K}$ when the crystal was warmed slowly. Several analytical tests were performed to ascertain the presence or absence of water in the sample. An infrared spectrum was taken on a Nujol mull, but nothing found could be attributed to water. The freezing temperature of a saturated aqueous $\mathrm{TIN}_{3}$ solution was determined with a Hewlett-Packard quartz thermometer as only $-0.03 \mathrm{~K}$, more than $0.4 \mathrm{~K}$ above the observed anomaly. Although the infrared spectrum taken on the calorimetric sample of $\mathrm{TIN}_{3}$ showed no evidence of water in the sample, the concentration of water may have been below detection limits. Recent neutron-diffraction experiments down to about $250 \mathrm{~K}$ show no evidence of a transition near $270 \mathrm{~K}^{(4)}$ The excess enthalpy (about $17.8 \mathrm{cal}_{\mathbf{t h}} \mathrm{mol}^{-1}$ ) in the region of this anomaly is, therefore, attributed to water, the mass fraction of which is estimated to be 0.001 based on the enthalpy of fusion of water. ${ }^{(22)}$

\section{IMPURITY ADJUSTMENT}

Adjustments for the small amount of water in the samples of $\mathrm{RbN}_{3}$ and $\mathrm{TIN}_{3}$ were made on the experimental heat capacity by the use of the heat capacity of pure water determined by Giauque and Stout. ${ }^{(24)}$ These corrections have been made on all $\mathrm{RbN}_{3}$ and $\mathrm{TIN}_{3}$ values in tables 5 and 6 , with the exception of the results taken in the vicinity of the fusion maxima.

TABLE 5. Thermophysical properties of $\mathrm{KN}_{3}, \mathrm{RbN}_{3}$, snd $\mathrm{CsN}_{3}$

$$
\left(\mathrm{cal}_{\mathrm{th}}=4.184 \mathrm{~J}\right)
$$

\begin{tabular}{|c|c|c|c|c|}
\hline$\frac{T}{\mathrm{~K}}$ & $\frac{C_{p}}{\mathrm{cal}_{\mathrm{th}} \mathrm{K}^{-1} \mathrm{~mol}^{-1}}$ & $\frac{S^{\circ}(T)-S^{\circ}(0)}{\mathrm{cal}_{\mathrm{th}} \mathrm{K}^{-1} \mathrm{~mol}^{-1}}$ & $\frac{H^{\circ}(T)-H^{\circ}(0)}{\mathrm{cal}_{\mathrm{th}} \mathrm{mol}^{-1}}$ & $\frac{-\left\{G^{\circ}(T)-H^{\circ}(0)\right\} / T}{\mathrm{cal}_{\mathrm{th}} \mathrm{K}^{-1} \mathrm{~mol}^{-1}}$ \\
\hline \multicolumn{5}{|c|}{$\mathrm{KN}_{3}$} \\
\hline 5 & 0.008 & $(0.003)$ & $(0.010)$ & $(0.001)$ \\
\hline 10 & 0.060 & 0.022 & 0.167 & 0.006 \\
\hline 15 & 0.220 & 0.071 & 0.798 & 0.018 \\
\hline 20 & 0.576 & 0.178 & 2.700 & 0.043 \\
\hline 25 & 1.444 & 0.364 & 6.916 & 0.088 \\
\hline 30 & 1.895 & 0.637 & 14.450 & 0.155 \\
\hline 35 & 2.771 & 0.994 & 26.078 & 0.249 \\
\hline 40 & 3.693 & 1.424 & 42.232 & 0.368 \\
\hline 45 & 4.620 & 1.912 & 63.02 & 0.512 \\
\hline 50 & 5.525 & 2.446 & 88.39 & 0.678 \\
\hline 60 & 7.198 & 3.605 & 152.19 & 1.068 \\
\hline 70 & 8.631 & 4.825 & 231.55 & 1.517 \\
\hline 80 & 9.816 & 6.058 & 323.98 & 2.008 \\
\hline 90 & 10.783 & 7.272 & 427.14 & 2.526 \\
\hline 100 & 11.577 & 8.450 & 539.1 & 3.059 \\
\hline 110 & 12.242 & 9.586 & 658.3 & 3.601 \\
\hline 120 & 12.817 & 10.676 & 783.6 & 4.146 \\
\hline 130 & 13.327 & 11.722 & 914.4 & 4.689 \\
\hline 140 & 13.791 & 12.727 & 1050.0 & 5.227 \\
\hline 150 & 14.218 & 13.694 & 1190.1 & 5.760 \\
\hline 160 & 14.614 & 14.624 & 1334.3 & 6.285 \\
\hline 170 & 14.979 & 15.52 & 1482.3 & 6.802 \\
\hline
\end{tabular}


LOW-TEMPERATURE HEAT CAPACITY OF KN $\mathrm{K}_{3}, \mathrm{RbN}_{3}, \operatorname{CsN}_{3}$, AND TIN $_{3}$

TABLE 5-continued

\begin{tabular}{|c|c|c|c|c|}
\hline $\begin{array}{l}T \\
\mathrm{~K}\end{array}$ & $\frac{C_{p}}{\text { cal }_{\text {th }} \mathbf{K}^{-1} \mathrm{~mol}^{-1}}$ & $\frac{S^{\circ}(T)-S^{\circ}(0)}{\mathrm{cal}_{\mathrm{th}} \mathbf{K}^{-1} \mathrm{~mol}^{-1}}$ & $\frac{H^{\circ}(T)-H^{\mathrm{c}}(0)}{\mathrm{cal}_{\mathrm{th}} \mathrm{mol}^{-1}}$ & $\frac{-\left\{G^{\circ}(T)-H^{\circ}(0)\right\} / T}{\mathrm{cal}_{\mathrm{th}} \mathrm{K}^{-1} \mathrm{~mol}^{-1}}$ \\
\hline 180 & 15.32 & 16.39 & 1633.7 & 7.310 \\
\hline 190 & 15.63 & 17.22 & 1788.5 & 7.810 \\
\hline 200 & 15.91 & 18.03 & 1946.2 & 8.301 \\
\hline 210 & 16.19 & 18.82 & 2106.7 & 8.783 \\
\hline 220 & 16.45 & 19.57 & 2269.9 & 9.257 \\
\hline 230 & 16.70 & 20.31 & 2435.7 & 9.721 \\
\hline 240 & 16.96 & 21.03 & 2604.0 & 10.178 \\
\hline 250 & 17.21 & 21.73 & 2774.9 & 10.626 \\
\hline 260 & 17.47 & 22.41 & 2948.3 & 11.066 \\
\hline 270 & 17.72 & 23.07 & 3124.2 & 11.498 \\
\hline 280 & 17.97 & 23.72 & 3302.7 & 11.923 \\
\hline 290 & 18.20 & 24.35 & 3483.6 & 12.341 \\
\hline 300 & 18.42 & 24.97 & 3666.7 & 12.751 \\
\hline 310 & 18.63 & 25.58 & 3851.9 & 13.156 \\
\hline 320 & 18.83 & 26.18 & 4039.2 & 13.553 \\
\hline 330 & 19.04 & 26.76 & 4228.6 & 13.945 \\
\hline 340 & 19.26 & 27.33 & 4420.1 & 14.330 \\
\hline 350 & 19.49 & 27.89 & 4613.9 & 14.709 \\
\hline 273.15 & 17.80 & 23.28 & 3180.2 & 11.633 \\
\hline 298.15 & 18.38 & 24.86 & 3632.6 & 12.676 \\
\hline \multicolumn{5}{|c|}{$\mathrm{RbN}_{3}$} \\
\hline 5 & 0.014 & $(0.007)$ & $(0.027)$ & $(0.002)$ \\
\hline 10 & 0.181 & 0.055 & 0.420 & 0.014 \\
\hline 15 & 0.673 & 0.210 & 2.402 & 0.049 \\
\hline 20 & 1.471 & 0.507 & 7.665 & 0.124 \\
\hline 25 & 2.432 & 0.936 & 17.377 & 0.241 \\
\hline 30 & 3.475 & 1.471 & 32.120 & 0.400 \\
\hline 35 & 4.551 & 2.088 & 52.19 & 0.596 \\
\hline 40 & 5.585 & 2.764 & 77.56 & 0.825 \\
\hline 45 & 6.569 & 3.479 & 107.97 & 1.080 \\
\hline 50 & 7.482 & 4.219 & 143.13 & 1.356 \\
\hline 60 & 9.072 & 5.728 & 226.16 & 1.959 \\
\hline 70 & 10.357 & 7.227 & 323.55 & 2.605 \\
\hline 80 & 11.381 & 8.679 & 432.43 & 3.274 \\
\hline 90 & 12.203 & 10.069 & 550.5 & 3.952 \\
\hline 100 & 12.878 & 11.391 & 676.0 & 4.631 \\
\hline 110 & 13.449 & 12.646 & 807.7 & 5.303 \\
\hline 120 & 13.945 & 13.837 & 944.7 & 5.965 \\
\hline 130 & 14.388 & 14.972 & 1086.4 & 6.614 \\
\hline 140 & 14.788 & 16.05 & 1232.3 & 7.250 \\
\hline 150 & 15.15 & 17.09 & 1382.1 & 7.872 \\
\hline 160 & 15.49 & 18.07 & 1535.3 & 8.479 \\
\hline 170 & 15.81 & 19.02 & 1691.8 & 9.071 \\
\hline 180 & 16.10 & 19.94 & 1851.4 & 9.650 \\
\hline 190 & 16.38 & 20.81 & 2013.8 & 10.214 \\
\hline 200 & 16.65 & 21.66 & 2179.0 & 10.765 \\
\hline 210 & 16.92 & 22.48 & 2346.9 & 11.304 \\
\hline 220 & 17.19 & 23.27 & 2517.4 & 11.830 \\
\hline 230 & 17.45 & 24.04 & 2690.6 & 12.344 \\
\hline 240 & 17.71 & 24.79 & 2866.4 & 12.847 \\
\hline 250 & 17.96 & 25.52 & 3044.8 & 13.340 \\
\hline 260 & 18.21 & 26.23 & 3225.6 & 13.822 \\
\hline 270 & 18.45 & 26.92 & 3408.9 & 14.294 \\
\hline
\end{tabular}


TABLE 5-continued

\begin{tabular}{|c|c|c|c|c|}
\hline$\frac{T}{\mathrm{~K}}$ & $\frac{C_{p}}{\mathrm{cal}_{\mathrm{th}} \mathbf{K}^{-1} \mathrm{~mol}^{-1}}$ & $\frac{S^{\circ}(T)-S^{\circ}(0)}{\mathrm{cal}_{\mathrm{th}} \mathrm{K}^{-1} \mathrm{~mol}^{-1}}$ & $\frac{H^{\circ}(T)-H^{\circ}(0)}{\mathrm{cal}_{\mathrm{th}} \mathrm{mol}^{-1}}$ & $\frac{-\left\{G^{\circ}(T)-H^{\circ}(0)\right\} / T}{\mathrm{cal}_{\mathrm{th}} \mathrm{K}^{-1} \mathrm{~mol}^{-1}}$ \\
\hline 280 & 18.68 & 27.59 & 3594.5 & 14.757 \\
\hline 290 & 18.91 & 28.25 & 3782.5 & 15.21 \\
\hline 300 & 19.14 & 28.90 & 3972.7 & 15.66 \\
\hline 310 & 19.37 & 29.53 & 4165.2 & 16.09 \\
\hline 320 & 19.59 & 30.15 & 4360.0 & 16.52 \\
\hline 330 & 19.82 & 30.76 & 4557.1 & 16.95 \\
\hline 340 & 20.04 & 31.35 & 4756.4 & 17.36 \\
\hline 350 & 20.25 & 31.93 & 4957.9 & 17.77 \\
\hline 273.15 & 18.52 & 27.13 & 3467.1 & 14.441 \\
\hline 298.15 & 19.09 & 28.78 & 3937.4 & 15.58 \\
\hline \multicolumn{5}{|c|}{$\mathrm{CsN}_{3}$} \\
\hline 5 & 0.051 & $(0.001)$ & $(0.003)$ & $(0.000)$ \\
\hline 10 & 0.564 & 0.161 & 1.303 & 0.031 \\
\hline 15 & 1.533 & 0.563 & 6.420 & 0.135 \\
\hline 20 & 2.656 & 1.157 & 16.874 & 0.313 \\
\hline 25 & 3.789 & 1.871 & 32.987 & 0.552 \\
\hline 30 & 4.926 & 2.662 & 54.77 & 0.837 \\
\hline 35 & 6.038 & 3.506 & 82.21 & 1.157 \\
\hline 40 & 7.062 & 4.380 & 115.00 & 1.505 \\
\hline 45 & 8.002 & 5.267 & 152.69 & 1.874 \\
\hline 50 & 8.858 & 6.155 & 194.88 & 2.258 \\
\hline 60 & 10.319 & 7.904 & 291.03 & 3.054 \\
\hline 70 & 11.477 & 9.585 & 400.24 & 3.868 \\
\hline 80 & 12.386 & 11.180 & 519.7 & 4.683 \\
\hline 90 & 13.102 & 12.681 & 647.3 & 5.489 \\
\hline 100 & 13.679 & 14.093 & 781.3 & 6.280 \\
\hline 110 & 14.161 & 15.42 & 920.6 & 7.051 \\
\hline 120 & 14.581 & 16.67 & 1064.3 & 7.801 \\
\hline 130 & 14.964 & 17.85 & 1212.1 & 8.529 \\
\hline 140 & 15.32 & 18.97 & 1363.5 & 9.235 \\
\hline 150 & 15.66 & 20.04 & 1518.4 & 9.921 \\
\hline 160 & 15.99 & 21.06 & 1676.7 & 10.585 \\
\hline 170 & 16.30 & 22.04 & 1838.2 & 11.231 \\
\hline 180 & 16.60 & 22.98 & 2002.7 & 11.858 \\
\hline 190 & 16.89 & 23.89 & 2170.1 & 12.467 \\
\hline 200 & 17.17 & 24.76 & 2340.4 & 13.060 \\
\hline 210 & 17.44 & 25.61 & 2513.4 & 13.638 \\
\hline 220 & 17.70 & 26.42 & 2689.1 & 14.201 \\
\hline 230 & 17.97 & 27.22 & 2867.4 & 14.749 \\
\hline 240 & 18.24 & 27.99 & 3048.4 & 15.28 \\
\hline 250 & 18.51 & 28.74 & 3232.2 & 15.81 \\
\hline 260 & 18.79 & 29.47 & 3418.6 & 16.32 \\
\hline 270 & 19.07 & 30.18 & 3608.0 & 16.82 \\
\hline 280 & 19.36 & 30.88 & 3800.1 & 17.31 \\
\hline 290 & 19.65 & 31.57 & 3995.2 & 17.79 \\
\hline 300 & 19.94 & 32.24 & 4193.2 & 18.26 \\
\hline 310 & 20.23 & 32.90 & 4394.0 & 18.72 \\
\hline 320 & 20.52 & 33,54 & 4597.8 & 19.17 \\
\hline 330 & 20.82 & 34.18 & 4804.5 & 19.62 \\
\hline 340 & 21.12 & 34.80 & 5014 & 20.06 \\
\hline 350 & 21.43 & 35.42 & 5227 & 20.49 \\
\hline 273.15 & 19.16 & 30.40 & 3668.2 & 16.98 \\
\hline 298.15 & 19.89 & 32.11 & 4156.3 & 18.17 \\
\hline
\end{tabular}


TABLE 6. Thermophysical properties of $\mathrm{TIN}_{3}$ $\left(\mathrm{cal}_{\mathrm{th}}=4.184 \mathrm{~J}\right)$

\begin{tabular}{|c|c|c|c|c|}
\hline$\frac{T}{\mathrm{~K}}$ & $\frac{C_{p}}{\mathrm{cal}_{\mathrm{th}} \mathrm{K}^{-1} \mathrm{~mol}^{-1}}$ & $\frac{S^{\circ}(T)-S^{\circ}(0)}{\mathrm{cal}_{\mathrm{th}} \mathrm{K}^{-1} \mathrm{~mol}^{-1}}$ & $\frac{H^{\circ}(T)-H^{\circ}(0)}{\mathrm{cal}_{\mathrm{tn}} \mathrm{mol}^{-1}}$ & $\frac{-\left\{G^{\circ}(T)-H^{\circ}(0)\right\} / T}{\mathrm{cal}_{\mathrm{th}} \mathrm{K}^{-1} \mathrm{~mol}^{-1}}$ \\
\hline 5 & 0.076 & $(0.033)$ & $(0.125)$ & $(0.008)$ \\
\hline 10 & 0.874 & 0.277 & 2.117 & 0.066 \\
\hline 15 & 2.073 & 0.856 & 9.457 & 0.226 \\
\hline 20 & 3.247 & 1.615 & 22.791 & 0.476 \\
\hline 25 & 4.333 & 2.458 & 41.779 & 0.787 \\
\hline 30 & 5.356 & 3.339 & 66.02 & 1.139 \\
\hline 35 & 6.350 & 4.240 & 95.30 & 1.517 \\
\hline 40 & 7.283 & 5.150 & 129.42 & 1.914 \\
\hline 45 & 8.122 & 6.057 & 167.96 & 2.324 \\
\hline 50 & 8.890 & 6.953 & 210.54 & 2.743 \\
\hline 60 & 10.206 & 8.694 & 306.23 & 3.591 \\
\hline 70 & 11.295 & 10.352 & 413.91 & 4.439 \\
\hline 80 & 12.195 & 11.921 & 531.5 & 5.277 \\
\hline 90 & 12.941 & 13.402 & 657.3 & 6.098 \\
\hline 100 & 13.561 & 14.798 & 789.9 & 6.899 \\
\hline 110 & 14.084 & 16.12 & 928.2 & 7.678 \\
\hline 120 & 14.530 & 17.36 & 1071.3 & 8.433 \\
\hline 130 & 14.919 & 18.54 & 1218.6 & 9.166 \\
\hline 140 & 15.27 & 19.66 & 1369.6 & 9.876 \\
\hline 150 & 15.59 & 20.72 & 1523.9 & 10.564 \\
\hline 160 & 15.89 & 21.74 & 1681.3 & 11.231 \\
\hline 170 & 16.19 & 22.71 & 1841.7 & 11.878 \\
\hline 180 & 16.49 & 23.64 & 2005.1 & 12.505 \\
\hline 190 & 16.78 & 24.54 & 2171.4 & 13.116 \\
\hline 200 & 17.08 & 25.41 & 2340.8 & 13.709 \\
\hline 210 & 17.39 & 26.25 & 2513.1 & 14.286 \\
\hline 220 & 17.70 & 27.07 & 2688.6 & 14.849 \\
\hline 230 & 18.02 & 27.86 & 2867.2 & 15.40 \\
\hline $233.00 \approx$ & 123.2 & & & \\
\hline $242.04^{a}$ & 164.1 & & & \\
\hline 250 & 18.11 & 29.90 & 3068.7 & 17.62 \\
\hline 260 & 18.40 & 30.62 & 3251.3 & 18.11 \\
\hline 270 & 18.63 & 31.32 & 3436.5 & 18.59 \\
\hline 280 & 18.85 & 32.00 & 3623.9 & 19.05 \\
\hline 290 & 19.07 & 32.66 & 3813.5 & 19.51 \\
\hline 300 & 19.30 & 33.31 & 4005.3 & 19.96 \\
\hline 310 & 19.54 & 33.95 & 4199.5 & 20.40 \\
\hline 320 & 19.80 & 34.57 & 4396.2 & 20.84 \\
\hline 330 & 20.06 & 35.19 & 4595.4 & 21.26 \\
\hline 340 & 20.32 & 35.79 & 4797.3 & 21.68 \\
\hline 350 & 20.57 & 36.38 & 5001.7 & 22.09 \\
\hline 273.15 & 18.70 & 31.53 & 3495.3 & 18.74 \\
\hline 298.15 & 19.25 & 33.19 & 3969.6 & 19.88 \\
\hline
\end{tabular}

a Peak of transition 


\section{THERMAL FUNCTIONS}

The experimental heat capacities in the non-transition regions were curve-fitted to polynomials in reduced temperature by the method of least-squares and then integrated to yield values of the thermal functions at regular temperature intervals presented in tables 5 and 6 . The thermodynamic functions have a probable error of less than 0.1 per cent above $50 \mathrm{~K}$. Below $5 \mathrm{~K}$, entropy and enthalpy increments were obtained from plots of $C_{p} / T$ against $T^{2}$. No adjustments for contributions due to isotopic mixing or nuclear spin were made; hence, the values tabulated are practical values for use in chemical thermodynamic calculations.

\section{Discussion}

\section{POTASSIUM, RUBIDIUM, AND CESIUM AZIDES}

That $\mathrm{KN}_{3}, \mathrm{RbN}_{3}$, and $\mathrm{CsN}_{3}$ have no low-temperature thermal anomalies in the heat capacity is shown in figure 1. Pistorius predicted low-temperature phase transitions for $\mathrm{RbN}_{3}$ and $\mathrm{CsN}_{3}{ }^{\left({ }^{8)}\right.}$ based on the assumption that $p(T)$ equilibrium lines could be extrapolated linearly to $101 \mathrm{kPa}$. These lines may be concave downwards so that the low-temperature phases of $\mathrm{RbN}_{3}$ and $\mathrm{CsN}_{3}$ would only be encountered at higher pressures. A similar prediction by White and Pistorius ${ }^{(25)}$ was made for $\mathrm{CsHF}_{2}$, but no thermal anomaly was detected below $300 \mathrm{~K}$ in heat-capacity measurements of $\mathrm{CsHF}_{2} \cdot{ }^{(2)}$ For $\mathrm{CsHF}_{2}$, as for $\mathrm{RbN}_{3}$ and $\mathrm{CsN}_{3}$, this phase transition would only be encountered at pressures higher than $100 \mathrm{kPa}$. An extrapolation similar to that for $\mathrm{CsHF}_{2}$ is not possible for $\mathrm{RbHF}_{2}$ because the phase diagram is incomplete.

Figure 4 illustrates the heat capacities of $\mathrm{KN}_{3}, \mathrm{RbN}_{3}$, and $\mathrm{CsN}_{3}$ and those of the respective $\mathrm{MHF}_{2}$ 's to facilitate comparison between the azides and the hydrogen difluorides. The similarities are to be expected since all the compounds consist of linear, symmetrical anions which belong to the same space group, I4/mcm- $\mathrm{D}_{4 \mathrm{~h}}^{1 \mathrm{~B}}$, and the contributions from internal frequencies of the anions are small. The only difference arises from the slightly larger molar masses of the azides relative to their respective hydrogen difluorides. Table 7 cites the entropies at $298.15 \mathrm{~K}$ for the azides and the corresponding hydrogen difluorides for further comparison.

\section{THALLIUM AZIDE}

Reproducibility of enthalpy increments over the transition region is shown in table 4 . The lattice heat capacity was deduced from graphical extrapolations of effective $\Theta_{D}$ 's against temperature into the transition region from above and below to get two values of $\Theta_{D}$ (lattice). These values of $\Theta_{D}$ (lattice) between 220 and $261 \mathrm{~K}$ were converted to lattice heat-capacity values extrapolated to $242 \mathrm{~K}$ to evaluate the excess enthalpy and entropy. From the difference between the integrated experimental heat-capacity curve and the estimated lattice contribution the total enthalpy and entropy of the transitions were obtained. The lattice enthalpy $\left\{H^{\circ}(261 \mathrm{~K})-H^{\circ}(220 \mathrm{~K})\right\}$ was determined to be $742.1 \mathrm{cal}_{\mathrm{th}} \mathrm{mol}^{-1}$. The resulting entropy and enthalpy increments of the transitions were found to be $\Delta S_{\mathrm{t}}=(0.89 \pm 0.01) \mathrm{cal}_{\mathrm{th}} \mathrm{K}^{-1} \mathrm{~mol}^{-1}$ and $\Delta H_{\mathrm{t}}=(195.9 \pm 2)$ $\mathrm{cal}_{\mathrm{th}} \mathrm{mol}^{-1}$. If a lower estimate of the lattice contribution had been chosen the lattice enthalpy $\left\{H^{\circ}(261 \mathrm{~K})-H^{\circ}(220 \mathrm{~K})\right\}$ would have been $738 \mathrm{cal}_{\mathrm{th}} \mathrm{mol}^{-1}$. The 


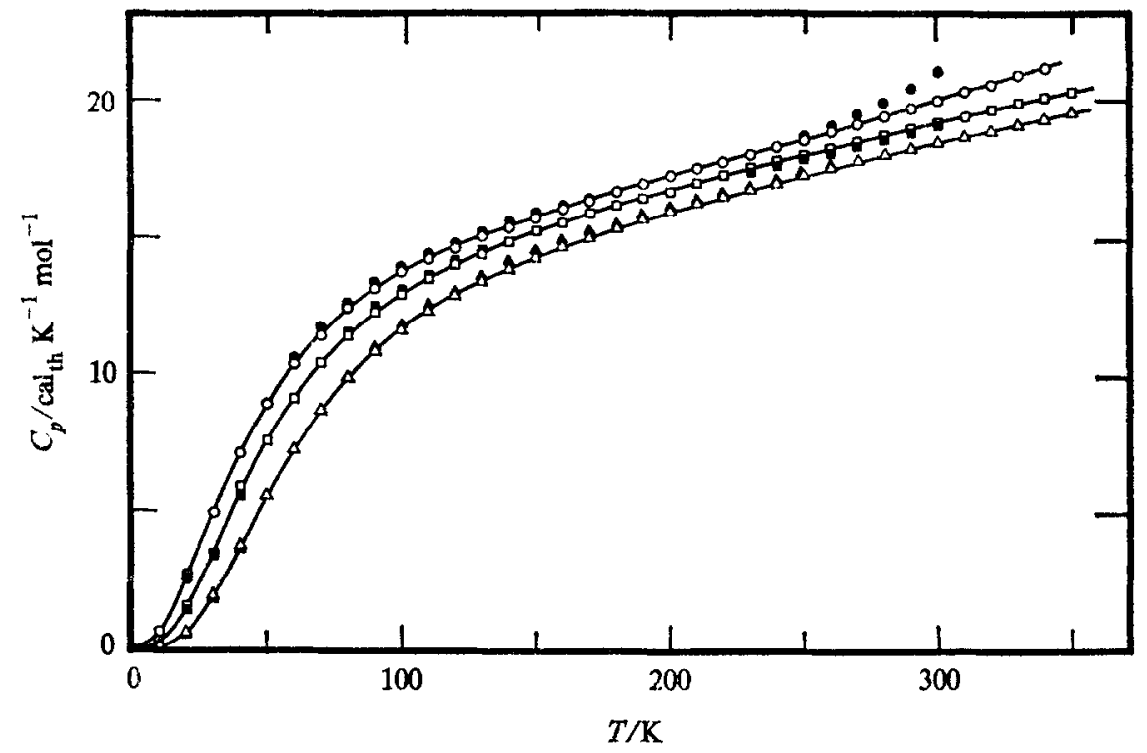

FIGURE 4. Smoothed heat capacities. $\triangle, \mathrm{KN}_{3} ; \boldsymbol{\Lambda}, \mathrm{KHF}_{3} ; \square, \mathrm{RbN}_{2} ; \mathbf{a}, \mathrm{RbHF}_{2} ; \mathrm{C}, \mathrm{CsN}_{3}$; - $\mathrm{CsHF}_{2}$. Data for the $\mathrm{MHF}_{2}$ compounds are from Burney and Westrum. ${ }^{(9)}$

TABLE 7. Entropies for the azides and hydrogen difluorides at $298.15 \mathrm{~K}$ $\left(\mathrm{cal}_{\mathrm{th}}=4.184 \mathrm{~J}\right)$

\begin{tabular}{llllll}
\hline \multicolumn{1}{c}{ Cation } & $\mathrm{Na}$ & $\mathrm{K}$ & $\mathrm{Rb}$ & $\mathrm{Cs}$ & Tl \\
\hline$S\left(\mathrm{MN}_{3}\right) / \mathrm{cal}_{\mathrm{th}} \mathrm{K}^{-1} \mathrm{~mol}^{-1}$ & $23.15^{a}$ & 24.86 & 28.78 & 32.11 & 33.19 \\
$S\left(\mathrm{MHF}_{2}\right) / \mathrm{cal}_{\mathrm{th}} \mathrm{K}^{-1} \mathrm{~mol}^{-1}$ & $21.73^{\circ}$ & $24.92^{\circ}$ & $28.70^{d}$ & $32.30^{d}$ & $34.92^{a}$ \\
\hline
\end{tabular}

\footnotetext{
${ }^{a}$ Carling and Westrum. ${ }^{(1)}{ }^{b}$ Burney and Westrum. ${ }^{(2)}{ }^{c}$ Westrum and Pitzer. ${ }^{(3)}{ }^{d}$ Burney and Westrum. ${ }^{(9)}$
}

resulting total entropy and enthalpy increments of the transitions would have been $\Delta S_{\mathrm{t}}=(0.91 \pm 0.01) \mathrm{cal}_{\mathrm{th}} \mathrm{K}^{-1} \mathrm{~mol}^{-1}$ and $\Delta H_{\mathrm{t}}=(200.5 \pm 2) \mathrm{cal}_{\mathrm{th}} \mathrm{mol}^{-1}$. Although the thermal properties of the components of the bifurcated anomaly cannot be resolved unambiguously, the apparent enthalpies and entropies associated with the lower peak are chosen as $(160 \pm 10) \mathrm{cal}_{\mathrm{th}} \mathrm{mol}^{-1}$ and $(0.71 \pm 0.05) \mathrm{cal}_{\text {th }} \mathrm{K}^{-1} \mathrm{~mol}^{-1}$ and with the upper $(40 \pm 10) \mathrm{cal}_{\mathrm{th}} \mathrm{mol}^{-1}$ and $(0.18 \pm 0.05) \mathrm{cal}_{\mathrm{th}} \mathrm{K}^{-1} \mathrm{~mol}^{-1}$, respectively.

Thermal effects in $\mathrm{TlN}_{3}$ have been studied by a variety of other methods also. A phase transition detected by d.t.a. at about $233 \mathrm{~K}$ has been reported. ${ }^{(3)}$ The infrared and Raman spectra indicated transition temperatures of $225 \mathrm{~K},{ }^{(11,26)}$ and $240 \mathrm{~K} .{ }^{(13)}$ A readily reversible transition at $(248 \pm 5) \mathrm{K}$ was found in the thermal expansion. ${ }^{(12)}$ Pressure equilibrium studies indicated a transition at $(193 \pm 30) \mathrm{K}^{\left({ }^{(8)}\right.}$ More recent d.t.a. work ${ }^{(14)}$ indicated a transition temperature of $(243.4 \pm 1.0) \mathrm{K}$. Although evidence for two closely-spaced transitions has not previously been reported, it seems plausible that the range in reported transition temperatures observed by different techniques may arise from one or other of the two transitions observed in the present research. 
The present work has yielded two transitions at 233 and $242 \mathrm{~K}$ and the following discussion is an attempt to elucidate the phase relations at high pressures for $\mathrm{TIN}_{3}$. Figure 5a shows the phase diagram based on the work of Pistorius. ${ }^{(14)}$ Recent attempts to resolve the problems in the light of the present results using d.t.a. at high pressures ${ }^{(27)}$ produced no additional information. A lack of reproducibility and the minute size of d.t.a. signals involved do not allow definite conclusions. Calorimetric studies under hydrostatic pressure conditions or high-pressure $\mathrm{X}$-ray diffraction studies would be most useful in resolving the situation.

The first possibility is that the two transitions present merge at higher pressures at a IV/III/II triple point, and the resulting phase boundary is the III/II boundary reported by Pistorius. Close examination of the determined slope of the III/II phase boundary shows this to be impossible. An added disqualification is the fact that the high volume change present at the III/II transition was not apparent in the transition from the II to IV phase. (Pistorius presents a detailed discussion of this.) ${ }^{(14)}$
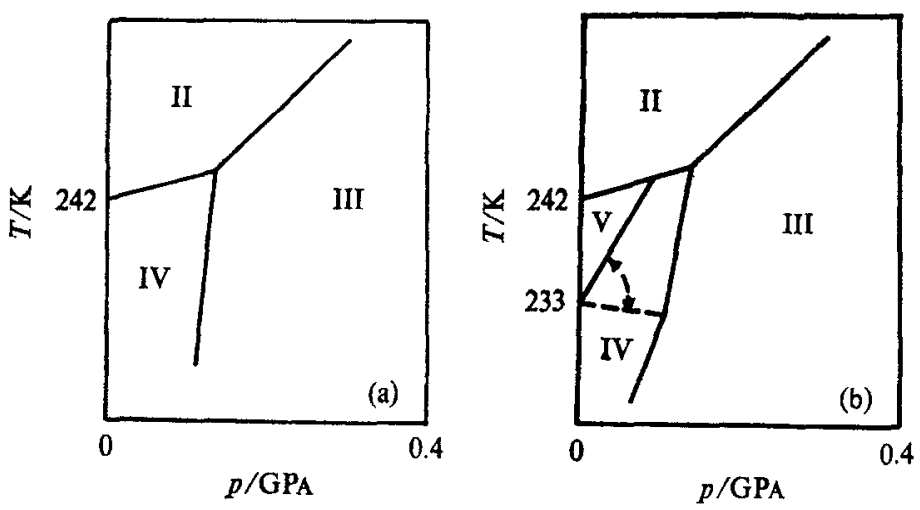

FIGURE 5. (a) Phase diagram at low pressures of $\mathrm{TIN}_{3}$ system according to Pistorius; (14) (b) possible revision proposed by Clark. ${ }^{(27)}$

There remain two possibilities which are both acceptable. Figure $5 \mathrm{~b}$ illustrates two possible slopes for a proposed IV/V phase boundary. The first one indicates a II/V/IV triple point at $0.15 \mathrm{GPa}$ near $244 \mathrm{~K}$. This value is chosen because of the disappearance of d.t.a. signals reported by Pistorius at this point. From this assumption it is possible to calculate the volume changes on the IV/V and V/II boundaries using the ClausiusClapeyron equation. These support the contention that the IV/III phase boundary carries most of the volume change associated with the III/II transition. The unique feature shown in figure $5 b$ is the existence of phase IV which is different from phase III. The existence of this phase has an added importance because it alters certain crystal-chemical arguments concerning the high-pressure phases $\mathrm{TIN}_{3}(\mathrm{III}), \mathrm{RbN}_{3}(\mathrm{III})$, and $\mathrm{CsN}_{3}$ (III). From previous data on all compounds mentioned, arguments have been advanced ${ }^{(14)}$ to show that these phases are probably isostructural and that they are related to the orthorhombic low-temperature phase ${ }^{(12.28)}$ found for $\mathrm{TIN}_{3}$ at atmospheric pressure. However, Raman data ${ }^{(29)}$ are said to provide evidence that 
these phases may, in fact, be different. The presence of the $\mathrm{TIN}_{3}$ IV/III phase boundary indicates that they are apparently so. The change from the tetragonal phase II through phase IV, and finally to phase III may be from an eight-fold to a 4-4 coordination with four long azide - metal bonds and four short azide - metal bonds. ${ }^{(12)}$ Such 4-4 coordination has been observed in $\mathrm{AgN}_{3},{ }^{(29)}$ with distortion which is a consequence of weak covalent bonding between the azide and the thallium ions. At higher temperature the thermal energy masks these distortions.

Since "aged" samples of $\mathrm{TIN}_{3}$ are reported to exhibit a transition at $225 \mathrm{~K}$, ${ }^{(13)}$ we note that the calorimetric sample employed in our endeavor was about six months old. Until details of this "time-dependent" transition are known,we consider the reported transition at $225 \mathrm{~K}$ to be that observed at $233 \mathrm{~K}$ in this study.

As in the comparison between $\mathrm{NaN}_{3}$ and $\mathrm{NaHF}_{2}$, ${ }^{(1)}$ it is noted that $\mathrm{TIN}_{3}$ and $\mathrm{TIHF}_{2}$ do not exhibit the same thermal behavior; the entropy at $298.15 \mathrm{~K}$ of $\mathrm{TlHF}_{2}$ is about 5 per cent larger than that of $\mathrm{TIN}_{3}$ (compare table 7). Hassel et al. ${ }^{(31)}$ performed analytical tests and an $\mathrm{X}$-ray diffraction analysis on a material initially identified as $\mathrm{TIHF}_{2}$. These analytical tests were later shown to be incorrect and the proper formula of the compound identified as $\mathrm{TlH}_{2} \mathrm{~F}_{3} \cdot \frac{1}{2} \mathrm{H}_{2} \mathrm{O} .^{(32)} \mathrm{Lee}^{\left({ }^{33)}\right.}$ suggested that the heatcapacity determinations by Burney ${ }^{(9)}$ might have been taken on a sample of the hemihydrate. If the calorimetric $\mathrm{TlHF}_{2}$ sample, whose heat capacity and composition were determined in this laboratory, was partially hydrated this might explain the seemingly large value of $S^{\circ}$ for thallium hydrogen difluoride at $298.15 \mathrm{~K}$. However, the analysis of the calorimetric sample of $\mathrm{TlHF}_{2}$ was in good agreement with theoretical. ${ }^{(9)}$ Evidence for hydration such as Hassel encountered was sought but not detected.

It seems probable that the difference in $S^{\circ}(298.15 \mathrm{~K})$ between $\mathrm{TIN}_{3}$ and $\mathrm{TIHF}_{2}$ is due to two factors. Firstly, $\mathrm{TIN}_{3}$ is orthorhombic below $233 \mathrm{~K}$, while $\mathrm{TlHF}_{2}$ is tetragonal below this temperature. Secondly, the heat capacity of $\mathrm{TlHF}_{2}$ begins to rise sharply near $300 \mathrm{~K}$ possibly as a premonitory effect-similar to that in $\mathrm{CsHF}_{2}$ (compare figure 4)-heralding the onset of a transition in $\mathrm{TlHF}_{2}$ noted in a recent report. ${ }^{(34)}$

\section{HEAT CAPACITY OF $\mathrm{KN}_{3}$ FROM SPECTRAL DATA}

Heat capacities at constant volume $\left(C_{V}\right)$ of these azides can be calculated from the acoustical and the optical spectral branches. The former contribution can be represented by a Debye term using a characteristic temperature determined from the heat capacity of the solid, and the latter by Einstein terms deduced from the frequencies. The tetragonal azides of this research have two formula units in each primitivecell, ${ }^{(35)}$ 21 optical and 3 acoustical modes. Although spectral data are available for all compounds in this research, only for $\mathrm{KN}_{3}$ and $\mathrm{TIN}_{3}$ are some thermal expansivities and compressibilities available.

For $\mathrm{KN}_{3}$, the $C_{V}$ calculated from spectral data and that calculated from our measured $C_{p}$ values with thermal expansivities and compressibilities are displayed in figure 6. As an alternative method for calculating $C_{V}$, the familiar relation represented by the first equality:

$$
C_{p}-C_{V}=T V \alpha^{2} / \kappa=A T C_{p}^{2}
$$


in which $V$ denotes molar volume, $\alpha$ thermal expansivity, and $\kappa$ isothermal compressibility may be recast as the term shown at the right (in which the symbol $A$ denotes $\left.V \alpha^{2} / \kappa C_{p}^{2}\right) . A$ remains approximately constant over significant temperature ranges. ${ }^{(36)}$ For $\mathrm{KN}_{3}$, a value of $A$ of $1.08 \times 10^{-5} \mathrm{~mol} \mathrm{cal}_{\mathrm{th}}^{-1}$ at $293 \mathrm{~K}$ was derived from the molar volume of $39.9 \mathrm{~cm}^{3} \mathrm{~mol}^{-1}$, a temperature-dependent thermal expansivity valid from 100 to $300 \mathrm{~K}$ (with a $293 \mathrm{~K}$ value of $144 \times 10^{-6} \mathrm{~K}^{-1}$, ${ }^{(37)}$ favored over an earlier value of $182 \times 10^{-6} \mathrm{~K}^{-1},(38)$, and a compressibility of $54.1 \mathrm{TPa}^{-1}$. For $\mathrm{TIN}_{3}$, the

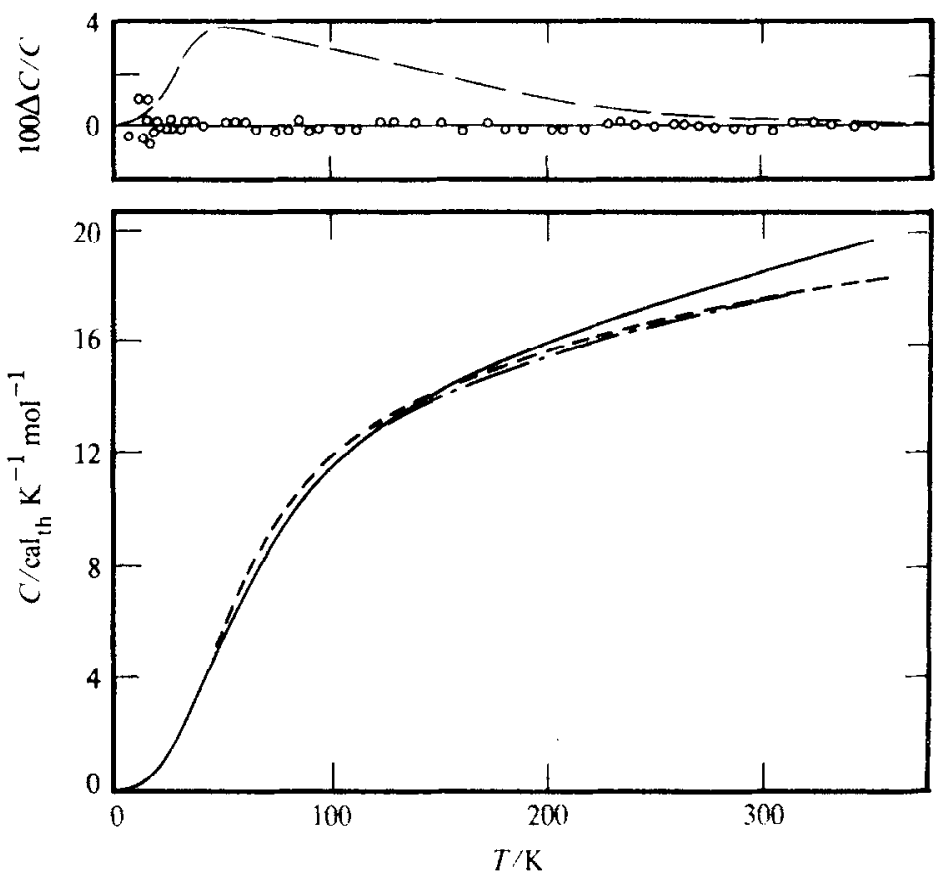

FIGURE 6. Comparison of heat capacity of $\mathrm{KN}_{3}$ evaluated by different methods.,$- C_{p}$ (expt); - - -,$C_{V}$ (calc. from spectral data); -.-, $C_{V}$ (calc. from $C_{p}$ (expt) adjusted for thermal expansivity and compressibility). The deviations, $O$, between the experimental $C_{p}$ points and the smoothed curve and -1 between the $C_{V}$ (from spectral data) and $C_{V}$ (calc. from $C_{p}$ ) are depicted in the upper portion of the figure.

value of $A$ of $1.21 \times 10^{-5} \mathrm{~mol} \mathrm{cal}_{\text {th }}^{-1}$ was deduced from $V=41.7 \mathrm{~cm}^{3} \mathrm{~mol}^{-1}, \alpha=$ $135 \times 10^{-6} \mathrm{~K}^{-1}$ below the $\mathrm{TIN}_{3}$ transition and $160 \times 10^{-6} \mathrm{~K}^{-1}$ above the transition, ${ }^{(12)}$ and $\kappa=46.3 \mathrm{TPa}^{-1}{ }^{(39)}$ Values of $C_{p}(\mathrm{expt})$ and of $C_{V}(\mathrm{calc}$.) from the expression above, displayed in figures 6 and 7 show good agreement with $C_{V}$ (spect.) at high temperatures but deviate as the temperature decreases. Such deviation has been observed in other solids also. ${ }^{(35)}$ Evaluation of $C_{V}$ from spectral data for $\mathrm{NaN}_{3}$ and the other two compounds of this study can be found elsewhere; (16) (however, only for $\mathrm{KN}_{3}$ and $\mathrm{TIN}_{3}$ are $\alpha$ and $\kappa$ available). For each of these azides the spectroscopic 

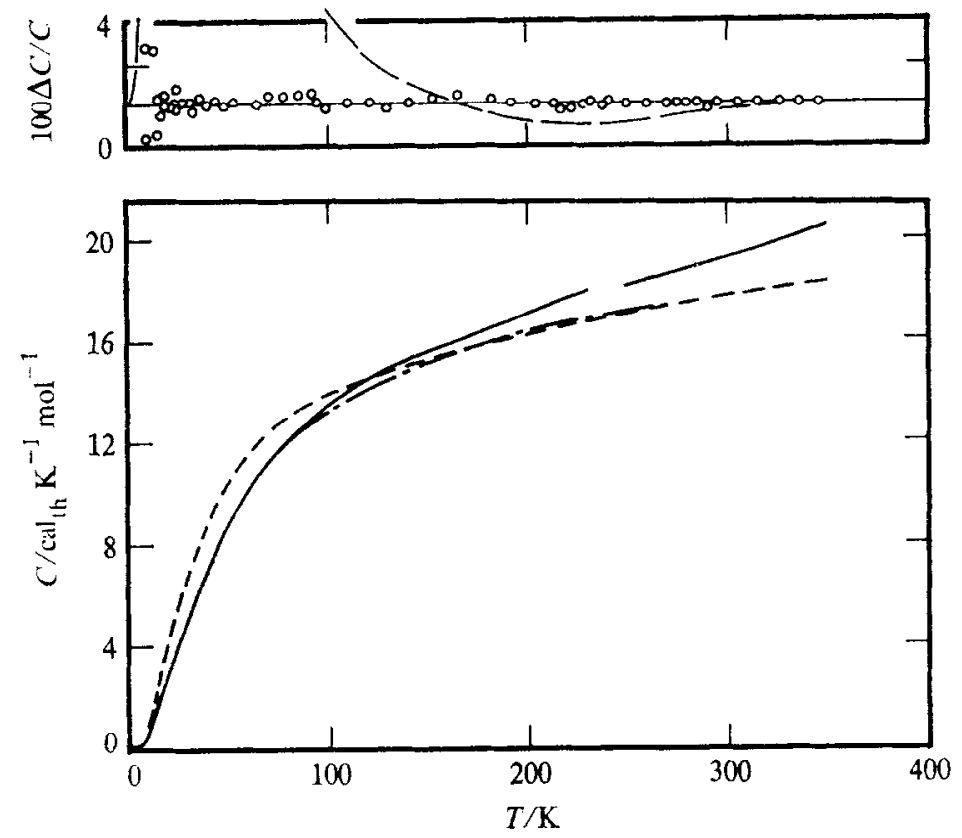

FIGURE 7. Comparison of heat capacity of $\mathrm{TIN}_{3}$ evaluated by different methods.,$- C_{p}(\mathrm{expt})$; - - - , $C_{V}$ (calc. from spectral data); -.-, $C_{V}$ (calc. from $C_{p}$ (expt) adjusted for thermal expansivity and compressibility). The deviations, $O$, between the experimental $C_{p}$ points and the smoothed curve and $-\ldots$, between the $C_{y}$ (from spectral data) and $C_{V}$ (calc. from $C_{p}$ ) are depicted in the upper portion of the figure.

$C_{V}$ exceeds the measured $C_{p}$ at lower temperatures. The relative success of the calculation for $\mathrm{KN}_{3}$ probably stems from the greater separation of optical and acoustical branches occasioned by the comparable masses of the two ions.

The authors express their appreciation to the Chemical Thermodynamics Program of the Chemistry Section, National Science Foundation for partial support of this endeavor and to E. I. Du Pont for fellowship support for R. W. Carling. We acknowledge the cooperation of A. J. Highe and C. G. Galeas, in the experimental measurements. Dr Brian Clark's helpful discussions are also acknowledged.

\section{REFERENCES}

1. Part I. Carling, R. W.; Westrum, E. F., Jr., J. Chem. Thermodynamics 1976, 8, 565.

2. Westrum, E. F., Jr.; Burney, G. J. Phys. Chem. 1961, 65, 344.

3. Westrum, E. F., Jr.; Pitzer, K. S. J. Am. Chem. Soc. 1949, 71, 1940.

4. Choi, C. S.; Prince, E. J. Chem. Phys. 1976, 64, 4510.

5. Mueller, H. J.; Joebstl, J. A. Z. Krist. 1965, 121, 385

6. Landee, C. P.; Westrum, E. F., Jr., unpublished results.

7. Evans, B. L.; Yoffe, A. D.; Gray, P. Chem. Revs. 1959, 59, 515.

8. Pistorius, C. W. F. T. J. Chem. Phys. 1969, 51, 2604.

9. Burney, G. A.; Westrum, E. F., Jr. J. Phys. Chem. 1961, 65, 349.

10. Kezer, O. F.; Rosenwasser, H. Nature 1966, $210,1354$.

11. Iqbal, Z.; Malhotra, M. L. J. Chem. Phys. 1972, 57, 2637. 
12. Mauer, F. A.; Hubbard, C. R.; Hahn, T. A. J. Chem. Phys. 1973, 59, 3770.

13. Iqbal, Z.; Christoe, C. W. Chem. Phys. Lett. 1974, 29, 623.

14. Pistorius, C. W. F. T. J. Chem. Phys. 1974, 60, 3720.

15. Suhrman, V. R.; Clusius, K. Z. Anorg. Chem. 1926, 152, 52.

16. Carling, R. W., Ph.D. thesis, The University of Michigan, Ann Arbor, Michigan, 1975 Diss. Abs. 1976, 36, 5056-B.

17. For detailed supplementary data concerning X-ray analysis of the samples used in this research, see NAPS document No. 02771 for 12 pages of supplementary material. Order from ASIS/ NAPS, c/o Microfiche Publications, 440 Park Avenue South, New York, New York 10016 U.S.A. Remit in advance for each NAPS accession number. Make checks payable to Microfiche Publications. Photocopies are \$5.00. Microfiche are \$3.00. Outside the U.S. and Canada postage is $\$ 2.00$ for a photocopy or $\$ 1.00$ for a fiche.

18. Clem, R. G.; Huffman, E. H. Anal. Chem. 1965, 37, 366.

19. Suzuki, S. J. Chem. Soc. Jpn., Pure Chem. Sect. 1952, 73, 150.

20. Vogel, A. I. A Text-Book of Inorganic Analysis, 3rd edition. Wiley: New York. 1961.

21. Westrum, E. F., Jr.; Furukawa, G. T.; McCullough, J. P. Adiabatic low-temperature calorimetry. In Experimental Thermodynamics vol. I. McCullough, J. P.; Scott, D. W.: editors. Butterworths: London. 1968.

22. Rossini, F. D.; Wagman, D. D.; Evans, W. H.; Levine, S.; Jaffe, I. Nat. Bur. Stand. Circular No. 500, U.S. Government Printing office, Washington, D.C. 1952.

23. Iqbal, Z. Personal communication. 1975.

24. Giauque, W. F.; Stout, J. W. J. Am. Chem. Soc. 1939, 58, 1144.

25. White, A. J. C.; Pistorius, C. W. F. T. J. Chem. Phys. 1972, 56, 4318.

26. Iqbal, Z. Advan. Raman Spec. 1972, 1, 188.

27. Clark, J. B. Personal communication. 1976.

28. Haase, O., unpublished results.

29. Christoe, C. W.; Iqbal, Z. Solid State Commun. 1974, 15, 859.

30. West, C. D. $Z$. Krist. 1936, 95, 421.

31. Hassel, V. O.; Kringstad, H. Z. Anorg. Allg. Chem. 1930, $191,36$.

32. Hassel, V. O.; Kringstad, H. Z. Anorg. Allg. Chem. 1932, 208, 382.

33. Lee, A. G. The Chemistry of Thallium. Elsevier: New York. 1971.

34. Zil'Berman, B. D.; Fedotova, T. D.; Gabuda, S. P. Zh. Strukt. Khim. 1976, 17, 273.

35. Hathaway, C. E.; Temple, P. A. Phys. Rev. B 1971, 3, 3497.

36. Zemansky, M. W. Heat and Thermodynamics. McGraw-Hill: New York. 1957.

37. Mauer, F. A.; Hahn, T. A. AlP Conference Proc. 1971, 3, 139.

38. Parsons, R. B.; Yoffe, A. D., Acta Cryst. 1966, $20,36$.

39. Weir, C. E.; Block, S.; Piermarini, G. J. J. Chem. Phys. 1970, 53, 4265. 